\title{
GURREN Symptom-based patient-reported outcomes in adults with eosinophilic esophagitis: value for treatment monitoring and randomized controlled trial design
}

\author{
Ekaterina Safroneeva $^{\mathrm{a}}$ and Alain M. Schoepfer ${ }^{\mathrm{b}}$
}

\begin{abstract}
Purpose of review
In adults with eosinophilic esophagitis (EoE), a chronic, inflammatory immune-mediated condition of the esophagus, both inflammation and fibrosis are likely associated with symptom generation. Therefore, assessing symptom-based patient-reported outcomes (PROs), defined by US Food and Drug Administration as 'any report of the status of a patient's health condition that comes directly from the patients, without interpretation of the patient's response by a clinician or anyone else', is important in the context of trials and observational studies of emerging therapies.
\end{abstract}

\begin{abstract}
Recent findings
For purposes of treatment monitoring, lack of symptoms does not predict the absence of biologic inflammation; hence, endoscopy with esophageal biopsies should be performed to check for residual inflammation. Lack of inflammation does not predict lack of symptoms, and the presence of subepithelial fibrosis cannot be excluded. No published instrument currently measures the frequency of dysphagia described all possible ways, strategies of living with this symptom and various pain types. In randomized controlled trials, in which symptom response was detected using validated PRO measures, only modest decreases in symptom scores were observed.
\end{abstract}

\section{Summary}

Accessing full EoE symptom spectrum and optimizing PRO measures remains a challenge that should be tackled to reliably assess response to existing and emerging therapies.

\section{Keywords}

patient-reported outcome measures, randomized controlled trials, symptoms, treatment monitoring

\section{INTRODUCTION}

Eosinophilic esophagitis (EoE) is a chronic, inflammatory immune-mediated condition of the esophagus, which is diagnosed based on the presence of symptoms of esophageal dysfunction and esophageal eosinophilia (threshold of $>15$ eosinophils per high-power field) [1]. With current incidence estimates from 5 to 10 cases per 100000 and current prevalence estimates from 0.5 to 1 case per 1000 , EoE is recognized as frequent cause of dysphagia (in up to $23 \%$ of all patients) and food bolus impactions (in up to $50 \%$ of all patients) $[1,2]$. It has been shown that, in adults with EoE, duration of untreated inflammation is positively associated with fibrosis and stricture formation $\left[3,4^{-"}, 5\right]$.

\section{SYMPTOM GENERATION IN ADULTS WITH EOSINOPHILIC ESOPHAGITIS}

It is likely that both inflammation and fibrosis are associated with symptom generation in adults with EoE. Endoscopic manifestations of fibrosis in EoE include rings and strictures. In a series of studies utilizing the functional luminal imaging probe

\footnotetext{
anstitute of Social and Preventive Medicine, University of Bern, Bern and ${ }^{b}$ Division of Gastroenterology and Hepatology, Centre Hospitalier Universitaire Vaudois/CHUV, University of Lausanne, Lausanne, Switzerland Correspondence to Ekaterina Safroneeva, $\mathrm{PhD}$, Institute of Social and Preventive Medicine, University of Bern, Mittelstrasse 43, 3012 Bern, Switzerland. E-mail: ekaterina.safroneeva@ispm.unibe.ch
}

Curr Opin Allergy Clin Immunol 2018, 18:000-000 DOI:10.1097/ACI.0000000000000514 


\section{KEY POINTS}

- Both inflammation and fibrosis are likely associated with symptom generation.

- Symptom-based PRO measures should be used for treatment monitoring.

- There is disconnect between severity of symptoms and biologic findings.

- Only modest decreases in dysphagia frequency-based PRO scores were observed in trials of anti-inflammatory therapies.

- Accessing full EoE symptom spectrum using PRO measures remains a challenge.

(examines regional variation of cross-sectional area, from which researchers calculate distensibility plateau considered to be a proxi for transmural remodeling/fibrosis), authors demonstrated that increasing ring grade as assessed by 'EoE endoscopic reference score (also stands for exudates, rings, edema, furrows, and strictures, EREFS)' grading and classification system was associated with lower esophageal distensibility plateau, and that distensibility plateau in patients, who presented with food impaction, was lower than in patients who did not [6-8]. Warners et al. [4"'] observed an association between stricture formation and bolus impactions in 106 patients. In the remaining 124 patients with bolus impactions, no stricture was observed [4"']. In International Eosinophilic Esophagitis Activity Index (EEsAI), patient global assessment of symptom severity was positively associated not only with increasing ring/stricture severity but also with increased severity of exudates assessed by EREFS [9]. Given that distensibility was not measured in Dutsch [4"'] and Swiss [9] studies, it is impossible to exclude the role of underlying fibrosis in the absence of obvious strictures in symptom generation. However, it is likely that extreme forms of inflammation are contributing to overall symptom severity. How exactly inflammation contributes to symptom generation remains to be elucidated. For example, no association between distensibility and either esophageal eosinophilia or exudates were observed $[6,7]$. With more than 100 patients enrolled (only five individuals with severe exudates), a jury is still out on whether inflammation decreases the crosssectional area of the esophagus to an extent that would cause symptoms. Recently, a study describing a case series of eight patients put forward another mechanism through which inflammation may cause symptoms. Upon injection of the solution containing trigger food antigen (so-called 'Esophageal Prick
Test'), authors observed almost immediate complete luminal obstruction that resolved after 3-10 min. In two patients, delayed responses were also observed upon second endoscopy. For the moment being, it is too early to say whether these spastic episodes might be a cause of bolus impactions and potentially other symptoms $\left[10^{\prime \prime}\right]$.

\section{PATIENTS-REPORTED OUTCOME MEASURES IN EOSINOPHILIC ESOPHAGITIS}

Given that the pathophysiologic hallmarks of EoE likely influence symptom severity, assessing symptoms appears to be paramount in daily clinical practice, observational studies and randomized clinical trials (RCTs). Although bolus impactions and dysphagia are well-documented symptoms of EoE, these and other less well-known EoE symptoms are mostly described from the perspective of a medical provider. However, in order to arrive at the patient-reported outcome (PRO), defined by US Food and Drug Administration (FDA) as 'any report of the status of a patient's health condition that comes directly from the patients, without interpretation of the patient's response by a clinician or anyone else', it is important to explore the way patients describe symptoms and other aspects of health [11]. In this review, we will focus on symptom-based PRO measures.

\section{SYMPTOMS OF EOSINOPHILIC ESOPHAGITIS AS PATIENTS DESCRIBE THEM}

Large qualitative studies exploring the full symptom spectrum in adults with EoE are scare; however, these are required by US FDA to inform the content of the PRO measure [11]. As a part of the EEsAI PRO study, the thematic content analyses of the patient interviews' $(n=24)$ and survey' $(n=135)$ transcripts were carried out [9]. As a part of development of Dysphagia Symptom Questionnaire (DSQ), 20 patients were interviewed to specifically describe their dysphagia-related concerns, and thematic analysis was carried out [12].

\section{Dysphagia}

Dysphagia is the most frequent symptom in adults with EoE. Three dysphagia-related themes were identified in EEsAI PRO study: descriptions and characteristics (frequency and location) of dysphagia; foods, pills and beverages causing dysphagia; and behavioral adaptations to living with dysphagia (two subthemes, including strategies for avoiding and dealing with impactions) (Fig. 1) [9]. During 


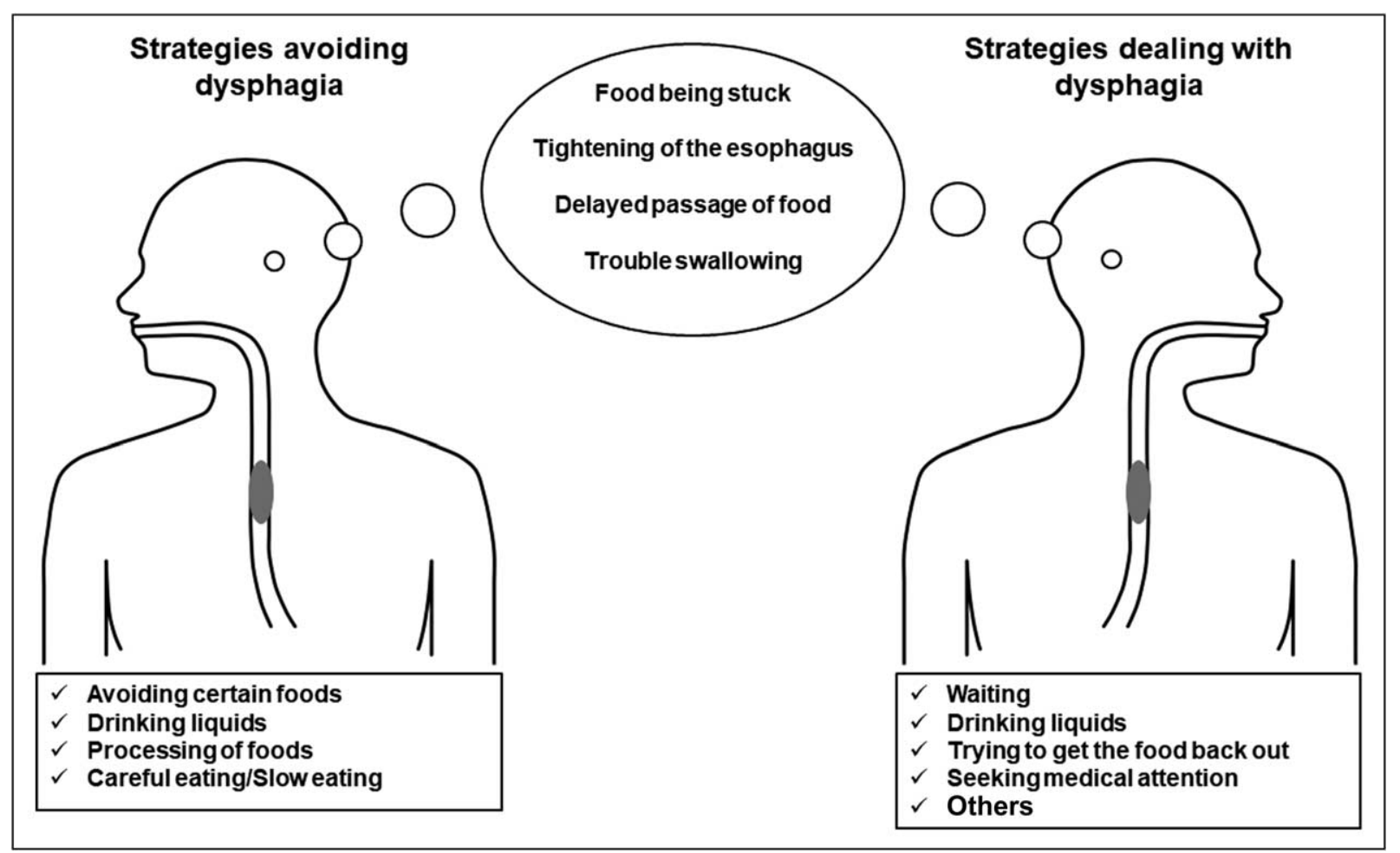

FIGURE 1. Dysphagia from patients' perspective.

DSQ development, descriptions of dysphagia and strategies dealing with dysphagia were explored [11]. Conceptually, both Swiss and US-based patients think of dysphagia in variety of ways, which included trouble swallowing (only detected using EEsAI PRO qualitative work), delayed passage of food, impactions (or food being stuck) and tightening of the esophagus $[9,11]$. At present, EEsAI PRO instrument assessed the frequency of dysphagia described as trouble swallowing, whereas furthest validated measure of dysphagia frequency, DSQ, assessed the frequency dysphagia described in two ways, namely food impactions (or food being stuck) and delayed food passage. Although neither instrument uses 'tightening of the esophagus' to describe dysphagia, patients' quotes supporting the use of this term have been demonstrated in articles describing the development of both instruments $[9,12]$. Although sensation of esophageal tightening might be important in the context of the immediate/delayed reactions to EoE food triggers [10"], patients used this description in the context of eating relatively dense foods, like meat. Strategies of avoiding and dealing with dysphagia episodes have been described during EEsAI PRO and DSQ development. Strategies of avoiding dysphagia episodes include avoiding certain mostly dense or very dry foods, modifying certain foods, trying to chew food more carefully and drinking copious amounts of liquids [9]. As for strategies dealing with dysphagia episodes, simply waiting, drinking liquids (which may or may not help the cause), trying to get the food back out (mostly described by Swiss German patients as vomiting and regurgitation and English-speaking US patients as vomiting, choking, coughing and gagging of the foods) and seeking medical attention to dislodge impacted food have been commonly described by the patients $[9,12]$. Adult EoE patients develop many strategies to deal with dysphagia episodes (including exercise and various relaxation techniques), and only the most common ones should be captured by dysphagiaassessing PRO instruments. Although both English and German belong to the same group of West Germanic languages, the way patients describe trying to get the food back out appears to be different, pointing to the importance of cross-cultural adaptations of the dysphagia-assessing PRO instruments. EEsAI PRO and DSQ instrument developers proceeded to include strategies of avoiding dysphagia (eating slowly, modifying and avoiding foods) and strategies of dealing with dysphagia (waiting, drinking liquids, vomiting, gagging, coughing and seeking medical attention to dislodge the impacted food) into their respective instruments $[9,12]$. In summary, PRO instruments published to date use different descriptions of dysphagia for assessing frequency of this symptom and examine different 
strategies of living with dysphagia. Future studies are needed to find out whether items containing all possible descriptions of dysphagia would be more sensitive in identifying patients with this symptom compared to items currently used in EEsAI PRO and DSQ, respectively. Similarly, the studies with headto-head comparisons of the extent to which various strategies of living with dysphagia contribute to overall patient symptom severity should be carried out.

\section{Others}

Although developers of DSQ were mostly interested in dysphagia, EEsAI PRO developers identified other important themes, including swallowing-associated and nonswallowing-associated pain. In nearly $40 \%$ of all patients, only food ingestion-associated themes emerged. In addition to already described dysphagia, the presence of pain when swallowing contributed in an important way to variation in patient global assessment of symptom severity and was included into the EEsAI PRO instrument. Although swallowing-associated pain and dysphagia are important to a large proportion of patients, other concerns relevant to patients, such as nonswallowing-associated symptoms, receive little attention [13] Evaluated as a part of the EEsAI PRO study, the presence of nonswallowing-associated pain was not associated with variation in patient global assessment of symptom severity. It remains to be determined whether pain intensity assessed using visual analogue scale were to perform better in explaining variation in patient global assessment of symptom severity. To simplify the symptom-based PRO instruments and diminish the burden on patients, as they complete these instruments on daily bases during RCT, the concerns falling outside of dysphagia assessment are often left behind. Other reasons why these concerns are overlooked include that they are less frequent than dysphagia, and that relatively small sample sizes of patients are often used to develop symptom-based PRO instruments in adults with EoE.

In summary, accessing a full spectrum of EoEassociated symptoms using PRO measures remains a challenge, and no published instrument currently measures the frequency of dysphagia described all possible ways, strategies of living with this symptom and various types of pain. Moving forward, existing and new instruments will continue to be validated and compared to arrive at optimal assessment of dysphagia and other EoE symptoms in adults. It may very well be that we will not need to describe dysphagia in four ways to assess its frequency, but we need the studies to show that.

\section{VALUE OF SYMPTOMS FOR TREATMENT MONITORING}

In adults with EoE, the relationship between symptoms, whether assessed by a validated PRO tool or not, and biologic findings continues to be a subject of much debate. The conflicting reports from relatively small RCT (old and new) have hinted that the relationship between symptoms and biologic findings, such as esophageal eosinophilia, may not be linear [9]. The data from relatively large $(n=269)$ for a rare disease observational, cross-sectional EEsAI study have demonstrated that EEsAI PRO score of 20 identified patients in endoscopic remission with $65.1 \%$ accuracy and histologic remission with 62.1\% accuracy, whereas EEsAI PRO score of 15 identified patients with both types of remission with $67.7 \%$ accuracy [14]. Reed et al. [15] approached a similar question from a different stand-point by examining the response to treatment in 62 consecutive patients. In patients who achieved histologic remission of less than 15 eosinophils per high-power field (eos/hpf), 70\% had an endoscopic response, $46 \%$ had a symptomatic response and $34 \%$ had both. In patients who achieved histologic remission of 0 eos/hpf posttreatment, $74 \%$ had an endoscopic response, $43 \%$ had symptomatic response and 38\% had both. As such, for the purposes of treatment monitoring, lack of symptoms, assessed by EEsAI PRO (7-day recall), does not predict the absence of biologic inflammation; hence, endoscopy with esophageal biopsies should be performed to find out whether residual inflammation is controlled by a given therapy. Naturally, these observations need to be confirmed using data from other large studies. In addition, lack of inflammation does not predict lack of symptoms, and the presence of subepithelial fibrosis cannot be excluded. When distensibility assessment becomes more main stream, this relationship between symptoms, inflammation and fibrosis needs to be re-examined.

\section{SYMPTOM-BASED PATIENT-REPORTED OUTCOMES INSTRUMENTS IN RANDOMIZED CONTROLLED TRIALS}

Although many investigator-initiated studies to assess efficacy of mostly corticosteroid therapies have been carried over the years, none of these studies used validated symptom-based PRO measures, and, hence, will not be discussed here. To date, the results of four phase II and phase III RCTs, in which symptom response was assessed either using DSQ (and similar instruments assessing the frequency of dysphagia) or EEsAI PRO, have been published in a form of full articles or abstracts presented during international meetings. It is also 
worth keeping in mind that certain phase II studies were not powered to detect symptom response. Daily assessment of symptoms in adults with EoE is one of the primary tenants of US FDA PRO Guidance for the Industry, so electronic DSQ was administered daily, whereas 7-day recall period EEsAI PRO was administered in either article or electronic form [11]. Phase II and III RCTs to assess the efficacy and safety of various anti-inflammatory therapies have been relatively small in comparison to those carried out in other chronic conditions.

Dellon et al. [16"'] reported the results of the phase II study on the efficacy and safety of 12-week $4 \mathrm{mg}$ budesonide suspension in 93 individuals with EoE (age ranged from 11 to 40 years of age). This budesonide formulation proved efficacious in reducing esophageal eosinophilia (mean decrease of $117 \mathrm{eos} / \mathrm{hpf}$ in drug treated vs. decrease of 17 eos/hpf in placebo-treated group, $P<.001)$ and DSQ score [mean decrease ( \pm standard deviation) of $14.3 \pm 13.0$ points in drug-treated group vs. decrease of $7.5 \pm 10.7$ points in placebo-treated group, $P=0.0096]$. The difference of less than 7 points separated drug-treated patients from those treated with placebo (DSQ ranges 0-84). In phase III study of budesonide orodispersible tablet, 88 adults with EoE were treated for 6 weeks with either $2 \mathrm{mg}$ budesonide orodispersible tablets $(\mathrm{n}=59)$ or placebo $(n=29)$ [17]. Once again, budesonide has proven to be efficacious in reducing esophageal eosinophilia (mean change of -226 eosinophils per $\mathrm{mm}^{2}$ in drug treated vs. -4 eosinophils per $\mathrm{mm}^{2}$, $P<0.0001)$ and EEsAI PRO score $(50.8 \%$ of patients achieved clinical remission defined as EEsAI $\mathrm{PRO} \leq 20$ in drug-treated group vs. $6.9 \%$ of patients in placebo-treated group, $P<0.0001$ ).

Efficacy and safety of RPC4046, anti-interleukin (IL)-13 antibody, was evaluated in a phase II trial in adults with EoE [18"]. Ninety-nine adults were randomized into one of three groups given RPC4046 (180 or $360 \mathrm{mg}$ ) or placebo once weekly for 16 weeks. At week 16, mean decrease of 90 or more eos/hpf in drug-treated groups was observed compared to an increase in $4.4 \mathrm{eos} / \mathrm{hpf}$ in a placebo group. At week 16 , mean decrease ( \pm standard deviation) in the dysphagia symptom diary (similar to DSQ instrument assessing dysphagia frequency) score was greater in the $360 \mathrm{mg}$ RPC4046 group compared with the placebo group $(13.3 \pm 15.3$ in drug-treated group vs. $6.41 \pm 15.40$ in a placebo-treated group, $P=0.0733)$. A trend for difference between mean change in EEsAI PRO in RPC4046 360 mg vs. placebo (difference of approximately 5-6 points between two groups, EEsAI PRO ranges 0-100) was observed. Finally, in a phase II study, the efficacy and safety of dupilumab, anti-IL-4R $\alpha$ monoclonal antibody, was evaluated in adults with EoE [19]. At week 12, a mean decrease of 94 eos/hpf in drug-treated groups was observed compared to decrease of 7 eos/hpf in the placebo group. At week 10, mean decrease in the EEsAI PRO score was greater in the dupilumab group compared to the placebo group (34.6 in drug-treated group vs. 11.3 in a placebo-treated group, $P=0.085$ ).

At times, it is difficult to compare these studies. Relatively severe patients were enrolled into all above-mentioned studies, and only modest (significant or trends) decreases in symptom scores were observed. Why this was the case remains to be determined. It could be that no instruments used all-encompassing dysphagia descriptions. It is also likely that, as patients start feeling better, they could be changing from softer to denser foods, while having the same dysphagia frequency. Whether patients explore denser food consistencies as these short-term studies progress has not been assessed. However, the data of the open-label extension (OLE) part of the RPC4046 study showed that EoE patients' dysphagia score continues to improve (the proportion of patients achieving symptomatic remission defined as EEsAI PRO score $\leq 20$ was $24.4 \%$ at OLE baseline, and $58.2 \%$ at week 52 with the caveat that there was no placebo arm) [20]. As phase II studies pave the way for large phase III RCT, these hypotheses will need to be explored.

\section{CONCLUSION}

EoE researchers, patients and other stakeholders will arrive at a core outcome set, including symptomsbased PRO, that will be used in RCT, observational studies and clinical practice [21]. However, assessing only dysphagia frequency in all types of studies might be too simplistic of an approach. As symptom-based and other PRO instruments get incorporated into telemedicine and e-health applications, we might make a distinction between daily dysphagia frequency assessment in RCT and more integrative approach toward symptom assessment during follow-up.

\section{Acknowledgements}

None.

\section{Financial support and sponsorship}

This work was supported by the grant from Swiss National Study Foundation (32003B_160115/1).

\section{Conflicts of interest}

E.S. received consulting fees from Aptalis Pharma, Inc., Celgene Corp., Novartis, AG and Regeneron Pharmaceuticals Inc. 
A.M.S. received consulting fees from Celgene Corp., Novartis, AG, Switzerland, Receptos, Inc. and Regeneron Pharmaceuticals, Inc.

A.M.S. received research grants from Adare Pharmaceuticals, Inc., AstraZeneca, AG, Switzerland, Aptalis Pharma, Inc., Celgene Corp., Dr Falk Pharma, GmbH, Germany, Glaxo Smith Kline, AG, Nestlé S. A., Switzerland, Novartis, AG, Switzerland, Receptos, Inc. and Regeneron Pharmaceuticals, Inc.

\section{REFERENCES AND RECOMMENDED READING}

Papers of particular interest, published within the annual period of review, have been highlighted as:

- of special interest

n. of outstanding interest

1. Lucendo AJ, Molina-Infante J, Arias Á, et al. Guidelines on eosinophilic esophagitis: evidence-based statements and recommendations for diagnosis and management in children and adults. United European Gastroenterol J 2017; 5:335-358.

2. Dellon ES, Hirano I. Epidemiology and natural history of eosinophilic esophagitis. Gastroenterology 2018; 154:319-332.

3. Schoepfer AM, Safroneeva E, Bussmann C, et al. Delay in diagnosis of eosinophilic esophagitis increases risk for stricture formation in a timedependent manner. Gastroenterology 2013; 145:1230-1236.

4. Warners MJ, Oude Nijhuis RAB, de Wijkerslooth LRH, et al. The natural

- course of eosinophilic esophagitis and long-term consequences of undiagnosed disease in a large cohort. Am J Gastroenterol 2018; 113:836-844. Confirms the findings by Schoepfer et al. [3] of a relationship between the length of diagnostic delay and stricture formation in relatively large population of adults with EoE.

5. Dellon ES, Kim HP, Sperry SL, et al. A phenotypic analysis shows eosinophilic esophagitis is a progressive fibrostenotic disease. Gastrointest Endosc 2014; 79:577-585

6. Chen JW, Pandolfino JE, Lin Z, et al. Severity of endoscopically identified esophageal rings correlates with reduced esophageal distensibility in eosinophilic esophagitis. Endoscopy 2016; 48:794-801.

7. Nicodème F, Hirano I, Chen J, et al. Esophageal distensibility as a measure of disease severity in patients with eosinophilic esophagitis. Clin Gastroenterol Hepatol 2013; 11:1101-1107.
8. Lin Z, Kahrilas $\mathrm{PJ}, \mathrm{Xiao} \mathrm{Y}$, et al. Functional luminal imaging probe topography: an improved method for characterizing esophageal distensibility in eosinophilic esophagitis. Therap Adv Gastroenterol 2013; 6:97-107.

9. Schoepfer AM, Straumann A, Panczak R, et al. Development and validation of a symptom-based activity index for adults with eosinophilic esophagitis. Gastroenterology 2014; 147:1255-1266.

10. Warners MJ, Terreehorst I, van den Wijngaard RM, et al. Abnormal responses

- to local esophageal food allergen injections in adult patients with eosinophilic esophagitis. Gastroenterology 2018; 154:57-60.

First article to describe the immediate response to esophageal allergen challenge in adults with EoE.

11. Available from https://www.fda.gov/downloads/drugs/guidances/ucm 1932 82.pdf. Last accessed on 7 November 2018.

12. Dellon ES, Irani AM, Hill MR, Hirano I. Development and field testing of a novel patient-reported outcome measure of dysphagia in patients with eosinophilic esophagitis. Aliment Pharmacol Ther 2013; 38:634-642.

13. Kahn J, Bussmann $C$, Beglinger $C$, etal. Exercise-induced chest pain: an atypical manifestation of eosinophilic esophagitis. Am J Med 2015; 128:196-199.

14. Safroneeva $E$, Straumann $A$, Coslovsky $M$, et al. Symptoms have modest accuracy in detecting endoscopic and histologic remission in adults with eosinophilic esophagitis. Gastroenterology 2016; 150:581-590.

15. Reed CC, Wolf WA, Cotton CC, et al. Optimal histologic cutpoints for treatment response in patients with eosinophilic esophagitis: analysis of data from a prospective cohort study. Clin Gastroenterol Hepatol 2018; 16:226-233.

16. Dellon ES, Katzka DA, Collins $\mathrm{MH}$, et al. Budesonide oral suspension

- improves symptomatic, endoscopic, and histologic parameters compared with placebo in patients with eosinophilic esophagitis. Gastroenterology $2017 ; 152: 776-786$.

The first trial to use validated symptom-based PRO measure.

17. Available from https://www.ema.europa.eu/documents/assessment-report/ jorveza-epar-public-assessment-report_en.pdf. Last accessed on 7 January 2019.

18. Hirano I, Collins MH, Assouline-Dayan Y, et al. RPC4046, a monoclonal antibody

- against IL13, reduces histologic and endoscopic activity in patients with eosinophilic esophagitis. Gastroenterology 2018; pii:S0016-5085(18)35208-9.

The second trial to use validated symptom-based PRO measures.

19. Hirano I, Dellon ES, Hamilton JD, et al. Dupilumab efficacy and safety in adult patients with active eosinophilic oesophagitis: a randomised double-blind placebo-controlled Phase 2 study. United European Gastroenterol J 2017; 5:1146.

20. Dellon ES, Collins $\mathrm{MH}$, Assouline-Dayan $\mathrm{Y}$, et al. Sustained long-term efficacy and safety of RPC4046, an anti-interleukin-13 monoclonal antibody, in patients with eosinophilic esophagitis: results from the open-label extension of the Heroes Study. Gastroenterology 2018; 154:S174-S175.

21. $M a C$, van Rhijn $B D$, Jairath $V$, et al. Heterogeneity in clinical, endoscopic, and histologic outcome measures and placebo response rates in clinical trials of eosinophilic esophagitis: a systematic review. Clin Gastroenterol Hepatol $2018 ; 16: 1714-1729$. 